\title{
Research progress of sorafenib in tumor therapy
}

\author{
Huijie Zhang, Weidong Guo \\ The Third Affiliated Hospital of Inner Mongolia Medical University, Baotou, China
}

Received: May 28, 2015

Accepted: August 4, $2015 \quad$ Online Published: September 10, 2015

DOI: $10.14725 /$ dcc.v2n3p41

URL: http://dx.doi.org/10.14725/dcc.v2n3p41

\begin{abstract}
Multi-kinase inhibitor Sola Fini (sorafenib), as the first approved multi-target agent, is a noval multi-target signal transduction inhibitors, which can inhibit the proliferation of tumor cells, prevent tumor angiogenesis and induce apoptosis of tumor cells. Studies have shown that Sola Fini imposes extensive antitumor activity on animal model of human tumor xenograft, such as mouse renal cell carcinoma, colon cancer, pancreatic cancer, thyroid cancer, non-small cell lung cancer, breast cancer and ovarian cancer model. In this paper, the mechanism of anti-tumor of sorafenib and the progress of tumor therapy are reviewed.
\end{abstract}

Key Words: Sorafenib, Targeted therapy, Tumor

In 1906, targeted therapy, first proposed by Enrilich, is a kind of therapy established on the basis of molecular biology of tumor. It refers to tumor tissue or cells with specific structural molecules by the use of certain specific binding with these target molecules antibodies and ligands, etc., to achieve direct or guided treatment by delivering a drug or substance that has a tumor-killing effect to the target site. Targeted therapy limits the therapeutic or pharmacological effects to specific target cells, tissues or organs, thereby improving efficacy and reducing adverse effects. Current studies suggest that the effect of targeted drugs in the treatment of malignant tumors is significantly better than traditional treatment, and toxic side effects are significantly reduced. Targeted drugs impose more "cure" effect than traditional treatments such as surgery, radiotherapy and chemotherapy, with strong specificity and significant effect, basically no damage to the normal tissue. ${ }^{[1]}$ With the development of modern medicine and the improvement of molecular biology technology, people gradually explore the pathogenesis of cancer from the molecular and gene levels. Tumor targeted therapy technology came into being then. The 5-year survival rate of cancer patients was improved by tumor targeted therapy and by delaying the progress of cancer. It has become a hot topic in the field of cancer therapy on how to improve the quality of life and prolong the survival of palliative care patients at the same time.

Sorafenib, the first approved targeted therapy, is an oral multi-kinase inhibitor, as well as a multiple target signal transduction inhibitor. It not only inhibits the proliferation of tumor cells by targeting the Raf kinase in Raf/MEK/ERK signal transduction pathway, but also induces apoptosis of tumor cells. ${ }^{[2-4]}$ It is mainly used in the treatment of advanced renal cell carcinoma for its good anti-tumor effect. ${ }^{[5]}$ It is also the first molecular targeted drug used in the treatment of liver cancer, and has been identified by the U.S. Food and Drug Administration (FDA). ${ }^{[6,7]}$

\section{Biological characteristics of sorafenib}

Preclinical studies have shown that sorafenib has a wide range of anti-tumor effects in digestive tract cancer, thyroid cancer, lung cancer, breast cancer, renal cell carcinoma, and so on. Researchers found that sorafenib could promote the specific phosphorylation of polyclonal anti-ERK1/2 an-

\footnotetext{
*Correspondence: Huijie Zhang; E-mail: zhanghuijie1210@hotmail.com; Address: The Third Affiliated Hospital of Inner Mongolia Medical University, Baotou, China.
} 
tibody and inhibit the test signal transduction pathway in tumor model using immunohistochemistry or Western-blot method. Wilhelm et al. ${ }^{[8]}$ found that sorafenib could inhibit tumor cells with K-ras and/or B-Raf mutations in the MAPK signaling system. Sun et al. ${ }^{[9]}$ also discovered the anti-tumor role of sorafenib by inhibiting autophagy in hepatoma cells. In addition, sorafenib was proved to restrain the angiogenesis of tumor by measuring the expression of CD31. In preclinical animal experiments, sorafenib showed better anti-tumor effect combined with irinotecan, cisplatin, paclitaxel, gemcitabine and other chemotherapy drugs with no increase of side effects.

Preclinical studies and clinical trials have demonstrated the dual anti-tumor effects of sorafenib. The growth of tumor depends on angiogenesis. VEGF and PDGF are the most important regulators of angiogenesis, and they can inhibit angiogenesis and tumor development related receptor tyrosine kinase activity, including PDGFR- $\beta$, FLT3, VEGFR-2, VEGFR-3 and C-KIT activity, blocking the angiogenesis and cutting off the nutrition supply for tumor cells, which indirectly inhibit tumor growth directly. ${ }^{[10]}$ On the other hand, the restrained activity of B-Raf and Raf1 inhibits Ras/Raf/MEK/ERK signal transduction pathway as well as the proliferation of tumor cells. In addition, sorafenib also suppresses related factors that contribute to the development and proliferation of tumor cells, such as FLT3 and C-KIT. Sorafenib finally achieves the outcome of blocking the proliferation of tumor cells by inhibiting the activity of these two factors.

\section{The mechanism of sorafenib}

Sorafenib is a novel multi-target anticancer drug and a small molecule kinase inhibitor. Studies in vivo and in vitro have shown that sorafenib could damage tumor microvessel by surpressing angiogenesis and cell proliferation, which caused tumor growth depression eventually. ${ }^{[1]}$ On the one hand, Sorafenib inhibits the activity of several kinds of receptor tyrosine kinase, including vascular endothelial growth factor receptor (VEGFR) 1, 2, 3, platelet-derived growth factor receptor (PDGFR), fibroblast growth factor receptor (FGFR), Ret, FLT and c-Kit, blocks tumor angiogenesis, reduces tumor cell nutrient supply, thereby inhibiting the proliferation and metastasis of tumor cells. ${ }^{[12]}$ Sorafenib, on the other hand, inhibits tumor cell proliferation through the inhibition of upstream KIT and FLT-3 receptor tyrosine kinase and downstream RAF-MEK-ERK pathway of serine threonine kinase. Its inhibition of tumor angiogenesis is accomplished by inhibiting upstream VEGFR-2 and PDGFR receptor tyrosine kinases, and also by inhibiting downstream Raf serine threonine kinases, thus effectively blocking the receptor tyrosine kinase signal transduction pathway, and giving full play to the anti-tumor effect of multi-channel and multi-channel. ${ }^{[13]}$ Chen et al. ${ }^{[14]}$ discov- ered that tumor blood vessels of patients taking sorafenib DSA contrast became sparse, which in a certain sense indicated that sorafenib could inhibit the expression of VEGF receptor activity, then further inhibiting tumor angiogenesis. Experiments in vitro certified that sorafenib can effectively inhibit Raf-1 activity at very low concentrations. The target involves Ras/Raf/MEK/MARK signal cascades, which suppresses the proliferation of tumor cells. Raf kinase is involved in the regulation of extracellular signal, the regulation of transcription factors, the inhibition of apoptosis and the regulation of cell differentiation. MEK is an important part of this signaling pathway, because it activates ERK in the cell signaling network. Identification of bispecific MEK and activation mechanism, greatly improves the accuracy of signal transduction, thus preventing ERK activation error. Liu et al. ${ }^{[4]}$ also confirmed that sorafenib could inhibit the activity of Raf kinase in hepatocellular carcinoma (HCC) cell line, and then block the MEK/ERK signal transduction pathway, and reduce the level of CyclinDl in HCC cell lines, thereby inhibiting the proliferation of tumor cells.

Apoptosis is also an important way of sorafenib to inhibit the proliferation of tumor cells. Sorafenib can prevent the proliferation of tumor cells and induce cell apoptosis in human HCC cell line HepG2 and PLC/PRF/5, and it also shows a wide range of anti-tumor activity in animal model. Sorafenib can induce the apoptosis of hepatoma cell line by blocking the Raf/MEK/ERK signaling pathway, decreasing the phosphorylation level of elF4E, and down regulating the expression of anti-apoptotic protein Md-l. Shi et al. ${ }^{[15]}$ found that sorafenib played an important role in promoting the apoptosis of HepG2 cells in a concentration-dependent manner. However, the inhibitory effect is held back along with the increase of drug concentration when the concentration increases to a certain value. ${ }^{[16]}$

\section{Sorafenib and tumor therapy}

\subsection{Thyroid carcinoma}

Surgery plus radioiodine therapy and endocrine suppression therapy is the standard treatment for the majority of differentiated thyroid cancer (DTC). Radioactive iodine has a good therapeutic effect on DTC with distant metastasis, whose 10 year survival rate is about 50\%. ${ }^{[17]}$ However, a small number of DTC patients are not sensitive to radioactive iodine treatment, and conventional radiotherapy and chemotherapy often show little effect. The prognosis of undifferentiated carcinoma is even worse. In a phase II clinical trial, 30 patients with advanced thyroid cancer patients took sorafenib (400 mg, twice daily), 4 weeks for a course. According to the evaluation criteria in solid tumors (RECIST), patients with partial remission (PR) accounted for $23 \%$ (7/30), maintained 18-84 weeks; patients with stable disease for 89 weeks or more accounted for 53\% (16/30); 
thyroglobulin levels decrease accounted for $95 \%(28 / 30){ }^{[18]}$ 32 cases of thyroid carcinoma were treated by sorafenib into the group for 26 weeks. PR was found in 8 cases, stable in 11 cases, progress in 7 cases according to the results of RECIST, CT, SPECT, TgA and TSH. The national comprehensive cancer network (NCCN) recommends sorafenib as the treatment of advanced thyroid cancer. Phase III clinical studies using sorafenib as a first-line drug for metastatic invasive thyroid cancer are in progress.

\subsection{Pulmonary carcinoma}

In recent years, due to the molecular targeted therapy, we have changed habitual thinking of the tumor and the strategy for the treatment of cancer. Epidermal growth factor receptor tyrosine kinase inhibitor (EGFR-TKIs) is particularly prominent in the treatment of advanced non-small cell lung cancer (NSCLC). During the past years, there was an increasing number of researches on the treatment of advanced NSCLC with EGFR-TKIs. Ultimately, patient will inevitably be resistant to TKIs regardless of the clinical outcomes. In that way, follow-up treatment almost becomes one of the hottest topics of lung cancer targeted therapy sessions. Clinical study of randomized double-blind, placebocontrolled, termination of a sorafenib three line single drug treatment of patients with NSCLC phase II showed that sorafenib significantly improved the outcomes for patients with PFS (3.6 months vs. 2.0 months, $p=.009$ ), and prolonged the OS (11.9 months vs. 9.0 months, $p=.18){ }^{[19]}$ In view of this phase II clinical study results, phase III clinical study by Global multicenter (MISSION study) in which the effect of sorafenib in the treatment of three/four line late or recurrence of metastatic NSCLC previously treated with TKIs was compared with placebo. The results are promising. Wang et al. discovered that common apoptosis associated with sorafenib was accompanied by upregulation of $\mathrm{Bax}, \mathrm{Bad}, \mathrm{Bcl}-\mathrm{xs}$, downregulation of $\mathrm{Bcl}$, and was closely related to the inhibition of ERK pathway in the study of of NSCLC (non small cell lung cancer) A549 cell line. ${ }^{[20]}$

\subsection{Digestive tumor}

\subsubsection{Hepatic carcinoma}

Since October 2007, the European Union EMEA, the United States FDA and SFDA in China have approved sorafenib as the first molecular targeted drug for clinical treatment of liver cancer. It has provided an effective treatment option for HCC patients. The affirmative efficacy of sorafenib in the treatment of liver cancer has been demonstrated by a large number of clinical trials. At the same time, sorafenib combined with chemotherapy drugs, as well as the combination of other intervention imposes considerable efficacy in the treatment of HCC, which brings a new hope for the treatment of advanced HCC patients. In a vitro study, Zhang et al. explored the killing effect of DC-CIK cells combined with sorafenib on human HCC line BEL-7402. ${ }^{[21]}$ The results showed that the inhibitory effect of sorafenib and DCCIK cells on liver cancer cells was enhanced in a certain concentration and target ratio. The highest killing rate was $72.24 \% \pm 2.42 \%$ at $20.8 \mu \mathrm{mol} / \mathrm{L}$ combined with DC-CIK target ratio of $40: 1$, which was higher than that in DC-CIK (1.8 times), sorafenib group (2.13 times) and CIK group (1.6 times) $(p<.01)$. The apoptotic rate of hepatocarcinoma cells was the highest in the combination of salafenib and DC-CIK cells $(77.36 \% \pm 1.92 \%, p<.05)$. It provides a new idea and theoretical basis for targeted therapy and immunotherapy of liver cancer. In the phase III clinical study, ${ }^{[22]} 602$ patients with advanced HCC were randomized to sarafenib or placebo control group, CR accounted for $43 \%$, control group for $32 \%$. The median OS for 10.7 months, the control group 7.9 months; disease progression time TTP 5.5 months, the control group 2.8 months.

\subsubsection{Gastric carcinoma}

Li et al. ${ }^{[23]}$ observed that sorafenib not only inhibited proliferation but also promoted apoptosis of human gastric cancer cell line SGC-7901. The inhibition rate was gradually increased with the prolongation of time and concentration of drug, appearing double effect relationship between dose and time $(p<.05)$. The P-ERK expression of SGC-7901 cells was significantly decreased by sorafenib $(p<.05)$. Clinical trials showed that sorafenib combined with traditional chemotherapy drugs significantly increased the anti-tumor effect, and reduced the dose and side effects of traditional chemotherapy drugs. The study on the inhibition effect of sorafenib on human gastric cancer cell line SGC-7901 will provide a more accurate theoretical basis for clinical treatment of gastric cancer, and expand its clinical application prospects.

\subsubsection{Pancreatic carcinoma}

Sorafenib, a multi-target tyrosine kinase inhibitor, has both anti-tumor and anti-angiogenesis effects. However, there are rare researches on its clinical application in pancreatic cancer. Clinical study at phase II showed that the performance of sorafenib as a first-line treatment for advanced pancreatic cancer was not satisfactory, whose median survival was 4 months, with 6-month survival rate of $23 \%$. $^{\text {[24] }}$

\subsubsection{Gallbladder carcinoma}

Targets of gallbladder cancer treatment with sorafenib include VEGFR-2/3, PDGFR- $\beta$, B-Raf and C-Raf. Sorafenib is effective for both wild and mutant B-Raf. At present, the use of sorafenib alone or combined with chemotherapy drugs such as gemcitabine, capecitabine for the treatment 
of gallbladder cancer has been employed. ${ }^{[25]}$ Furthermore, there were some reports regarding the application of VEGF antibody bevacizumab and sorafenib for the treatment of biliary tract cancer. Though the effect is obvious, the exact efficacy needs to be further verified. ${ }^{[26]}$

\section{Renal carcinoma}

Phase II clinical study of Akaza ${ }^{[27]}$ indicated the effect in 131 patients who received sustained treatment of sorafenib. Based on the RECIST evaluation criteria, the degree of tumor shrinkage showed 19 patients (14.7\%) with PR, 93 patients $(72.1 \%)$ with stable disease (SD), and 103 patients $(79.8 \%)$ with different degrees of shrinkage. The median progression-free survival (PFS) for all patients was 32 weeks. Six months with no progression-free survival rate showed SD 59\% vs. PR 88\%. In the Phase III clinical trial of Escudier et al., ${ }^{[28]} 903$ patients with advanced renal cell carcinoma were randomized to a sorafenib treatment and placebo control group. The results showed that ORR 9.7\% (CR 0.2\%, PR 9.5\%), control group ORR 1.8\% (CR 0, PR $1.8 \%$ ); SD 73.8\%, control group 52.9\%; median PFS 172 d, control group $85 \mathrm{~d}$.

Wang et al. ${ }^{[29]}$ investigated the killing effect of G250-DCCIK cells combined with sorafenib on renal carcinoma cells. When the target efficiency ratio is 20:1, the tumor killing rate of sorafenib was $58.4 \%$, sorafenib+CIK was $67.3 \%$, sorafenib+DC-CIK was $77.7 \%$, sorafenib+DC-CIK-G250 was $95.2 \%$. The killing effect of G250-DC-CIK cells combined with sorafenib on renal cell carcinoma was significantly better than that in other groups. Studies have shown that immunotherapy combined with drug targeted therapy not only directly inhibits tumor cells, but also indirectly kills tumor cells by regulating the immune system. Immunotherapy combined with drug targeted therapy is a new method for the treatment of renal cell carcinoma.

\section{Ovarian carcinoma}

Ovarian cancer patients are relatively sensitive to chemotherapy. However, due to the toxic side effects of chemotherapy, it cannot be used for a long time, which is one of the important reasons that led to the lower survival rate of ovarian cancer for years. Drug targeted therapy imposes no serious side effects to blood, liver and kidney system. It may be used as a maintenance therapy as well as consolidation therapy to achieve long-term survival of ovarian cancer patients. Matei et al. ${ }^{[30]}$ observed platinum-resistant recurrent ovarian cancer patients taking sorafenib in 59 clinical trial cases, and found that the median progression-free survival of more than 6 months accounted for $24 \%$, patients with PR accounted for $3.4 \%$, SD accounted for $33.9 \%$, and disease progress accounted for $50.8 \%$. The obvious side effects included skin rash, hand foot syndrome, metabolic abnormalities, gastrointestinal symptoms, heart and lung toxicity. The research results indicated the certain effect of sorafenib on the treatment of ovarian cancer with greater side reaction. In other words, the molecular targeted drugs in the treatment of ovarian cancer were not satisfactory. Liu et al. ${ }^{[31]}$ discovered that the effect of sorafenib combined with compound canth capsule in the treatment of ovarian cancer was $83.3 \%$, stable rate was $16.7 \%$ with no progress in 0 cases, while the effect of sorafenib monotherapy alone in the treatment of ovarian cancer was $60 \%$, stable rate $40 \%$ with no progress in 0 cases. The overall efficacy of the two groups was statistically significant $(p<.05)$. Adverse reactions included decreased hemoglobin, white blood cells, peripheral neuritis and alopecia without severe hepatorenal dysfunction. It turned out that the effect of oral administration of sorafenib in combination of Fufangbanmao Capsule was better and safer than that of single use of sorafenib in the treatment of ovarian cancer.

The greatest advantage of sorafenib, a molecular targeted drug, is to kill tumor cells selectively, and impose less damage to normal cells, which has a clinical application prospect in tumor treatment. However, there are few clinical trials in large samples. The detection of target expression and mutation before treatment, and the choice of individual drug therapy need to be further studied and explored. In addition, the occurrence, development, invasion and metastasis of tumor are attributed to multiple factors and abnormal genes. The treatment of cutting off one target is not comprehensive since signal transduction in cells is a multi-factor, complex, crossover protein network system.

Therefore, to find effective new therapeutic targets, multimolecular and multi-pathway targeted drug combination therapy, signal transduction pathway design, and combination of different molecular targeted drugs would be the development direction of tumor molecular targeted therapy. At present, the basic research and clinical trials have been under way. We are confident that in the near future, there will be more and more effective drugs to the target molecule and the scheme used for the treatment of tumors, which will bring greater benefits to the patients.

\section{Conflicts of Interest Disclosure}

The authors have no conflict of interest related to this article. 


\section{References}

[1] Vogelstein B, Kinzler KW. Cancer genes and the pathways they control. Nat Med. 2004; 10(8): 789-799. PMid: 15286780. https: //doi.org/10.1038/nm1087

[2] Flaherty KT. Sorafenib: delivering atargeted drug to the right targets. Expea Rev Anticaneer Ther. 2007; 7(5): 617-626. PMid: 17492926. https://doi.org/10.1586/14737140.7.5.617

[3] Hampton T. Cancer drug trials show modest benefit: drugs taget liver, gastric, head and neck caners. JAMA. 2007; 298(3): 273 275. PMid: 17635880. https://doi.org/10.1001/jama. 298 3.273

[4] Liu L, Cao Y, Chen C, et al. Sorafenib blocks the RAF/MEK/ERK pathway, inhibits tumor anglogenesis, and induces tumor cell apoptcsis in hepatocellular carcinoma model PLC/PRW/5. Cancer Res. 2006; 66(24): 11851-11858. PMid: 17178882. https://doi . org/10.1158/0008-5472. CAN-06-1377

[5] RatainM J, Eisen T, Stadler WM, et al. Phase II placebo-controlled randomized discontinuation trial of sorafenib in patients with metastatirenal cell carcinoma. J Clin Onco. 2006; 24(16): 2505-2512. PMid: 16636341. https://doi.org/10.1200/JC0.2005.03. 6723

[6] Abou-Alfa GK, Schwartz L, Ricci S, et al. Phase II study of sorafenib in patients with advanced hepatocellular carcinoma. J Clin Onco. 2006; 24(26): 4293-4300. PMid: 16908937. https://doi. org/10.1200/JC0.2005.01.3441

[7] Llovet JM, Ricci S, Mazzaferro V, et al. Sorafenib in advanced hepatocellular carcinom. The New England J Medicine. 2008; 359(4): 378-390. PMid: 18650514. https ://doi.org/10.1056/ NEJMoa0708857

[8] Wilhelm SM, Carter C, Tang L, et al. BAY 43-9006 exhibits broad Spectrum oral antitumor activity and targets the RAS/RAF/MEK/ERK pathway and receptor tyrosine kinases involved in tumor progression and angiogenesis. Cancer Res. 2004; 64(19): 7099-7109. PMid: 15466206. https://doi.org/10. 1158/0008-5472. CAN-04-1443

[9] Sun GW, Qiu WH, Shi MM, et al. Sola Feeney's inhibition of autophagy in hepatoma cell line. Surgical Theory and Practice. 2010; 15(1): 51-55.

[10] Strumberg D. Preclinical and clinical development of the oral multikinase inhibitor sorafenib in cancer treatment. Drugs Today (Barc). 2005; 41(12): 773-784. PMid: 16474853. https://doi.org/10. 1358/dot.2005.41.12.937959

[11] Wilhelm SM, Adnane L, Newell P, et al. Preclinical overview of sorafenib, a multikinase inhibitor that targets both Raf and VEGF and PDGF receptor tyrosine kinase signaling. Mol Cancer. 2008; 7(10): 3129-3140. PMid: 18852116. https://doi.org/ 10.1158/1535-7163.MCT-08-0013

[12] Chen KF, Chen HL, Tai WT, et al. Activation of phosphatidylinositol 3-kinase/Akt signaling pathway mediates acquired resistence to sorafenib in hepatocellular carcinoma. J Pharmacol Exp Ther. 2011; 337(1): 155-161. PMid: 21205925. https://doi.org/10.1124/ jpet. 110.175786

[13] Zhou KL, Zhu ZW. Clinical observation of therapeutic effects of sorafenib and biochemotherapy on metastatic renal cell carcinoma. China Journal of Modern Medicine. 2011; 21(1): 149-151.

[14] Chen SX, Chen J, Wei XI, et al. Transcatheter arterial chemoembolization combined with molecule-targeted sorafenib for the treatment of advanced hepatocelluar carcinoma: observation of its longterm efficacy. Journal of Interventional Radiology. 2011; 20(12): 958-960.
[15] Shi WH, Bian YH, Song XH, et al. Coadministration of Sorafenib with Adriamycin Inhibits Cell Proliferation in Hepatocellular Carcinoma Cells HepG2. Progress in Modern Biomedicine. 2011.

[16] Chaparro M, González ML, Trapero-Marugán M, et al. Review article: harmacological therapy for hepatocellular carcinoma with sorafenib and other oral agents. Aliment Pharmacol Ther. 2008; 28(1112): 1269-1277. PMid: 18808443. https://doi.org/10.1111/ j.1365-2036.2008.03857.x

[17] Durante C, Haddy N, Baudin E, et al. Long-term outcome of 444 patients with distant metastases from papillary and follicular thyroid carcinoma: benefits and limits of radioiodine therapy. J Clin Endocrinol Metab. 2006; 91(8): 2892-2899. PMid: 16684830. https : //doi.org/10.1210/jc.2005-2838

[18] Gupta-Abramson V, Troxel AB, Nellore A, et al. Phase II trial of sorafenib in advanced thyroid cancer. J Clin Oncol. 2008; 26(29): 4714-4719. PMid: 18541894. https://doi.org/10.1200/JCO. 2008. 16. 3279

[19] Schiller JH, Lee JW, Hanna NH, et al. A randomized discontinuation phase II study of sorafenib versus placebo in patients with non-small cell lung cancer who have failed at least two prior chemotherapy regimens: E2501. J Clin Oncol. 2008; 26(15): 431-436. https: //doi.org/10.1200/jco.2008.26.15_suppl.8014

[20] Wang B, Feng Y, Song X. et al. Involvement of ERK, Bcl-2 family and caspase 3 in recombinant human activaton A-indued apoptosis in A549. Toxicology. 2009 Apr 28; 258(2-3): 176-183. PMid: 19428937. https://doi.org/10.1016/j.tox.2009.01.023

[21] Zhang D, He JT. In vitro cytotox icity effects of cocultured DC-C IK cells combined with sorafenib against hepa to cellular carcinoma. Chinese Journal of Cellular \& Molecular Immunology. 2011; 27(6): 664.

[22] Chen ZP, Wang DL. Advances in clinical application of tumor molecular targeted therapies. Journal of Regional Anatomy and Operative Surgery. 2012; 21(2): 186-188.

[23] Li DY, Li LQ. Effect of Sola Feeney on proliferation, apoptosis and expression of P-ERK in human gastric cancer cell. Chinese Journal of General Surgery. 2011; 20(4): 367-371.

[24] Lou WH. Targeted therapy of pancreatic cancer. Chinese Journal of Practical Surgery. 2010; 30(7): 539-542.

[25] Bengala C, Bertolini F, Malavasi N, et al. Sorafenib in patients with advanced biliary tract carcinoma: a phase II trial. Br J Cancer. 2010; 102(1): 68-72. PMid: 19935794. https://doi.org/10.1038/ sj.bjc. 6605458

[26] Zhu AX, Hong TS, Hezel AF, et al. Current management of gallbladder carcinoma. Oncologist. 2010; 15(2): 168-181. PMid: 20147507. https://doi.org/10.1634/theoncologist.2009-0302

[27] Akaza H, Nsito S, Tsukamoto T, et al. Uncontrolled Confirmatory Trial of Single-Agent Sordenib in Japanese Patients with Advanced Renal Cell Carcinoma. Eur Uml Suppl. 2007; 6(2): 237. https://doi.org/10.1016/S1569-9056(07)60854-9

[28] Mori Y, Cai K, Cheng Y, et al. A Genome-Wide Search Identifies Epigenetic Silencing of Somatostatin. Tachykinin-1, and 5 Other Genes in Colon Cancer. Gastroenterology. 2006; 131(3): 797808. PMid: 16952549. https://doi.org/10.1053/j.gastro. 2006.06.006

[29] Wang ZY, Sun T, Ming MA. The effects of G250-DC-CIK cells combined with sorafenib on renal tumor cell. Chinese Journal of Biochemical Pharmaceutics. 2011; 6(3): 59-94.

[30] Matei D, Sill MW, Lankes HA, et al. Activity of sorafenib in recurrent ovarian cancer and primary peritoneal carcinomatosis: a gynecologic oncology group trial. J Clin Oncol. 2011; 29: 69-75. PMid: 21098323. https://doi.org/10.1200/JC0.2009.26.7856

[31] Liu JQ, Zheng Y, Yang Y, et al. Clinical Effect Analysis of Sorafenib Combined with Compound Banmao Capsule in the Treatment of Ovarian Cancer. Acta Chinese Medicine \& Pharmacology. 2011. 